\title{
Reduction of Portion Size and Eating Rate Is Associated with BMI-SDS Reduction in Overweight and Obese Children and Adolescents: Results on Eating and Nutrition Behaviour from the Observational KgAS Study
}

\author{
Gabriel Torbahna, b, c Ines Gellhaus ${ }^{d}$ Benjamin Koch d, e \\ Rüdiger von Kries ${ }^{a}$ Viola Obermeier ${ }^{a}$ Reinhard W. Holl ${ }^{f}$ Katharina Fink $^{f}$ \\ Andreas van Egmond-Fröhlich ${ }^{g}$ \\ ${ }^{a}$ Institute of Social Paediatrics and Adolescent Medicine, LMU Munich, Munich, Germany; \\ ${ }^{b}$ Institute for Medical Informatics, Biometry and Epidemiology, LMU Munich, Munich, \\ Germany; ${ }^{C}$ AdieuPositas, Ambulante Therapie für Kinder und Jugendliche, Munich, \\ Germany; ${ }^{d}$ Konsensusgruppe Adipositasschulung für Kinder und Jugendliche e.V. (KgAS), \\ Paderborn, Germany; ${ }^{e}$ Zentrum für Adipositasschulung Bremen-Stadt (ZABS), Bremen, \\ Germany; ${ }^{f}$ Institute for Epidemiology and Medical Biometry, ZIBMT, University of UIm, \\ Ulm, Germany; ${ }^{9}$ G. v. Preyer'sches Kinderspital SMZ-Süd, Vienna, Austria
}

\section{Keywords}

Childhood obesity · Adolescence obesity · Conservative treatment · Portion size - Eating rate

\section{Abstract}

Objective: Treatment of paediatric obesity focuses on changes of nutrition and eating behaviour and physical activity. The evaluation of the patient education programme by KgAS was utilised to analyse the association of changes of portion size, eating rate and dietary habits with BMI-SDS reductions. Methods: Patients $(n=297)$ were examined at the beginning and at the end of treatment and after 1-year follow-up at different out-patient centres. Their parents completed questionnaires including estimation of children's portion size, eating rate and frequency of food intake. Associations of 1- and 2-year changes in BMI-SDS and behaviour were calculated for patients with complete data in BMI-SDS, portion size, eating rate, frequency of green, yellow and red food intake $(n=131)$ by multiple linear regression models. Results: Significant changes were found in the desired direction for BMI-SDS, portion size, eating rate and the intake of unfavourable red food items both after 1 and 2 years as well as for the consumption of favourable green food items after 1 year. Significant positive associa- 
Torbahn et al.: Reduction of Portion Size and Eating Rate Is Associated with BMI-SDS Reduction in Overweight and Obese Children and Adolescents: Results on Eating and Nutrition Behaviour from the Observational KgAS Study

tions with BMI-SDS reduction after 1 and 2 years were detected for portion size (Cohen's $\mathrm{f}^{2}$ 0.13 and 0.09 ) and eating rate (Cohen's $f^{2} 0.20$ and 0.10 ), respectively. Conclusion: Reduced portion sizes and eating rates are associated with BMI-SDS reduction after 1 and 2 years. These findings suggest to focus on appropriate portion sizes and reduced eating rates in patient education programmes.

(C) 2017 The Author(s)

Published by S. Karger GmbH, Freiburg

\section{Introduction}

Childhood obesity represents a known risk factor for non-communicable diseases and one of the greatest health problems in western countries [1]. The high rate of children with persistent obesity up to adolescence and adulthood underlines the importance of prevention and treatment programmes during childhood [2].

The first KiGGS survey which is representative for the German population between 2 and 17 years revealed a prevalence of $8.7 \%$ and $6.3 \%$ for overweight and obesity in this age group [3]. In a follow-up survey, Germans between 11 and 17 years of age show overweight and obesity in $8.9 \%$ and $10 \%$ of this population, respectively, compared to $9.9 \%$ and $8.9 \%$ in the KiGGS baseline survey [4].

Multidisciplinary and multimodal cognitive behavioural group interventions form an important element of disease management programmes for children and adolescents in Germany. Recent systematic reviews and meta-analyses, however, showed limited effectiveness on weight loss [5-7].

The nutritional modules comprise practical and theoretical training units addressing dietary selection on the one hand and eating behaviour (e.g. eating rate, portion size necessary for satiety) on the other hand. Physical activity and exercise programmes showed significant BMI reduction in trials including overweight and obese children and adolescents with a moderate quality of evidence [8].

Furthermore, the construct of weight change is complex [9]. Weight changes are due to the integral of energy balance over time. The energy balance is defined as the difference of energy intake (from food and beverages) and energy expenditure (mainly resting energy and activity energy) [10]. Excessive cumulative energy intake appears to be the main contributor to weight status in children and adults on the basis of a general lack of physical activity. Energy intake is influenced by meal frequency, volume and energy density. The major causative role attributed to excessive energy density for the obesity epidemic has been questioned lately [11]. On the other hand, over the last years portion sizes (e.g. in fast food restaurants or at home) and package sizes have increased, and research increasingly focused on this topic $[12,13]$. As fast food restaurants serve large portions of energy-dense foods and exposure to a large portion size induces a higher eating rate and bite size, the contribution to energy intake is multiplied [12-16].

These findings from cross-sectional studies should be tested for effectiveness in real-life treatment programmes. Therefore, this research paper reports the changes in nutrition and eating behaviour and the effect of a reduced portion size and eating rate on BMI-SDS reduction.

\section{Patients and Methods}

Study Design

The KgAS (Konsensusgruppe Adipositasschulung für Kinder und Jugendliche e.V.) programme represents a guideline-orientated behaviour modification out-patient treatment for overweight and obese children and adolescents [17]. The KgAS study was conducted as an observational, prospective intervention (before- 
Torbahn et al.: Reduction of Portion Size and Eating Rate Is Associated with BMI-SDS Reduction in Overweight and Obese Children and Adolescents: Results on Eating and Nutrition Behaviour from the Observational KgAS Study

after) study with out-patients enrolled from August 2009 until February 2012. Patients have been treated at 14 centres in Germany (Augsburg, Bremen, Buchholz, Düsseldorf, Fürth, Hamburg, Homburg, Cologne, Lindau, Munich, Oberstaufen, Osnabrück, Paderborn, Würzburg) based on the KgAS protocol [18] which have been derived and further developed from the manual focussing on treating overweight and/or obese children and adolescents [19]. Measurements were conducted at the start of treatment (baseline; T0), at the end of treatment (1 year after baseline; T1) and after a follow-up of 1 year (2 years after baseline; T2).

\section{Intervention}

The intervention consisted of an out-patient multidisciplinary patient education programme. The modules provided medical background (comprising 3 units (of 45 min each) for patients and 3 units for parents), nutrition (21 units for patients and 14 units for parents) and exercise programmes (76 units for patients and 22 units for parents), psycho-social behaviour education (17 units for patients and 11 units for parents) in separate and common units. The nutrition module comprised theoretical (e.g. choice and ingredients of food based on the nutrition pyramid) and practical units (e.g. purchasing and preparing of food). Attention was given to eating behaviour (represented by portion size and eating rate) and nutrition behaviour (choice of food).

\section{Patients}

Patients were included if they were between 8 and 16 years of age as well as diagnosed as overweight or obese. According to the German national guideline, overweight and obesity were defined as BMI $\geq 90$ th and 97th BMI percentile, respectively [16]. Additionally, at least one questionnaire at baseline, completed by a parent or patient, was required for inclusion.

\section{Outcomes}

Weight and height of patients were assessed by paediatricians at the out-patient centres or at practices. According to patient's sex and age a BMI-standardised deviation score (BMI-SDS) was calculated according to the LMS method of Cole and Green 1992 [20]. Therefore, we used the reference LMS values published by Kromeyer-Hauschild et al. 2015 [21]. A negative value of the 1-year (T1-T0) and 2-year (T2-T0) BMI-SDS change indicated BMI-SDS reduction.

\section{Exposures}

All exposure variables were assessed via questionnaires handed out to parents.

Patient's eating rate was evaluated using the subscale 'eating rate' of the FKE-KJ questionnaire [22]. The mean for the Likert scale items: 'child eats fast, faster than his or her friends', 'consumes meals uninterrupted', 'chews slowly' (inverted) and 'eats slowly in terms of laying down it's fork or knife' (inverted) was calculated and standardised to a range of 0-100. This scale has a Cronbach's $\alpha=0.77$ [23].

Based on a food frequency list (FFQ), the frequency of child's average weekly consumption of the items was calculated from a Likert scale: 'never', '1-3 times monthly', '1-3 times weekly', '4-6 times weekly', '1-2 times daily' and '3-5 times daily' (range 0-28). The subscales 'healthy food intake' corresponding to green food items in the traffic light diet (fruits, vegetables, salads, whole-grain bread), 'indifferent food intake' (yellow food items: cheese, milk, quark, buttermilk and meat) and 'problematic food intake' (red food items in the traffic light diet: sweets, snacks, fast food, sausages, sweetened beverages, ice tea, cacao and fruit juices) were calculated by the respective mean [23].

The portion size was assessed on a linear scale from 1 to 7 . The parents were asked to estimate how much delicious pasta their child would eat if unattended. They could choose from six pictures of linearly ascending portions of pasta on plates using an item of the YANA-C computer-based pictorial instrument to assess nutritional intake in adolescents [24]. A 7th option was given as the largest portion size plus an extra helping. The pasta meal was chosen as it is broadly known and liked by children and adolescents [25].

Changes of portion size, eating rate and the intake of green, yellow and red food after 1 and 2 years were regarded as exposures.

\section{Confounders}

Patients' age and sex (assessed in patient questionnaires at baseline), the socio-economic status (SES) and migration background were defined as confounders.

The SES was assessed in the baseline parental questionnaire. According to Winkler and Stolzenberg [26], the SES is composed of the 'family income' as well as the higher value of maternal and paternal 'highest qualification in school and job' and 'highest job status'. Index values could range from 3-21. A range of 3-8 
Torbahn et al.: Reduction of Portion Size and Eating Rate Is Associated with BMI-SDS Reduction in Overweight and Obese Children and Adolescents: Results on Eating and Nutrition Behaviour from the Observational KgAS Study

was defined as 'low SES', a range of 9-14 as 'middle SES', and a range of 15-21 was classified as 'high SES' [27].

Migration background was assessed in the patient's questionnaire at baseline. According to the classification of the BZgA observational study, at least two out of three family members (patient, mother and father) had to be born abroad [28].

$\Delta$ variables (difference between measurement points of respective variables) associated with the primary exposures and the outcome $(\mathrm{p}<0.1)$ were regarded as potential confounders. Confounding was defined as a change in estimate (regression coefficient b) of $>10 \%$.

\section{Statistical Analyses}

All analyses were restricted to patients with complete data in BMI-SDS, portion size, eating rate as well as frequency of green, yellow and red food intake. If not stated otherwise, statistical tests were conducted on a significance level of $\mathrm{p}<0.05$.

Tests for normality were conducted using Q-Q plots and histograms. Significant differences in baseline characteristics between patients with complete and incomplete data were tested using the Student's t-test for independent samples for normally distributed continuous variables. For non-normally distributed variables we applied the Mann-Whitney U-test. The chi-square test was utilised for tests between categorical or nominal variables.

The change of variables was calculated by the difference between their measurement points (T1-T0 and T2-T0 for 1- and 2-year change). To test for differences between measurement points, the Wilcoxon-signed rank test or the t-test for dependent samples was used. Statements on effect sizes of 1- and 2-year changes were calculated using the formula of Glass' $\Delta$ with the standard deviation of the respective baseline value as reference:

Glass' $\Delta=\frac{\overline{x_{1}}-\overline{x_{2}}}{s_{2}}$

$\left(\mathrm{x}_{1}\right.$ : mean value of variable at $\mathrm{T} 2 ; \mathrm{x}_{2}$ : mean value of variable at baseline; $\mathrm{s}_{2}$ : standard deviation from variable at baseline) [29].

Associations between reduction of portion size, eating rate and 1- and 2-year BMI-SDS reduction were calculated by multiple linear regression. Correlations between all variables were considered. The selection of the final regression model was performed by forward selection. Additionally to the $\Delta$ value of BMI-SDS as the target variable and the explanatory variable (respective exposure, e.g. 1-year change of eating rate), the minimal model consisted of the baseline values of BMI-SDS and the respective explanatory variable as well as the confounding variables age, sex, SES and migration background. All confounders and independently significant $\Delta$ variables ascertained in the study were added to the full model. Due to a high correlation between the respective $\Delta$ variables of eating rate and portion size, we calculated separate regression models for the association between change of portion size or eating rate and 1- and 2-year BMI-SDS reduction. To test for possible interactions, we conducted all regressions with respective interaction terms. For significant regression coefficients in the final models, effect sizes were calculated as Cohen's $\mathrm{f}^{2}$ [30]. This effect size was derived from Cohen's d for multiple regression. It is calculated as

$$
f^{2}=\frac{R_{A B}^{2}-R_{A}^{2}}{1-R_{A B}^{2}}
$$

$\left(\mathrm{R}^{2}{ }_{\mathrm{A}}\right.$ : the variance accounted for $\left(=\mathrm{R}^{2}\right)$ by one (or more) independent variable $(\mathrm{s})$ of interest $\left.={ }_{\mathrm{A}}\right) ; \mathrm{R}^{2}{ }_{\mathrm{AB}}$ : the combination of variance accounted for by ${ }_{A}$ and another independent variable(s) $\left(={ }_{B}\right)$. Effect sizes of 0.02 indicate small effects, 0.15 medium effects and 0.35 indicate large effects. All statistical analyses were performed using R version 3.2.2 [31].

\section{Results}

297 patients met the inclusion criteria and were enrolled in the study. 34 patients discontinued therapy. After 1 year of follow-up, information regarding BMI-SDS and questionnaires could be obtained from 175 patients which corresponds to a loss to follow-up of 33.46\%. Complete data was available for 131 patients (44.11\%). Figure 1 shows the study flow chart. 
Torbahn et al.: Reduction of Portion Size and Eating Rate Is Associated with BMI-SDS Reduction in Overweight and Obese Children and Adolescents: Results on Eating and Nutrition Behaviour from the Observational KgAS Study

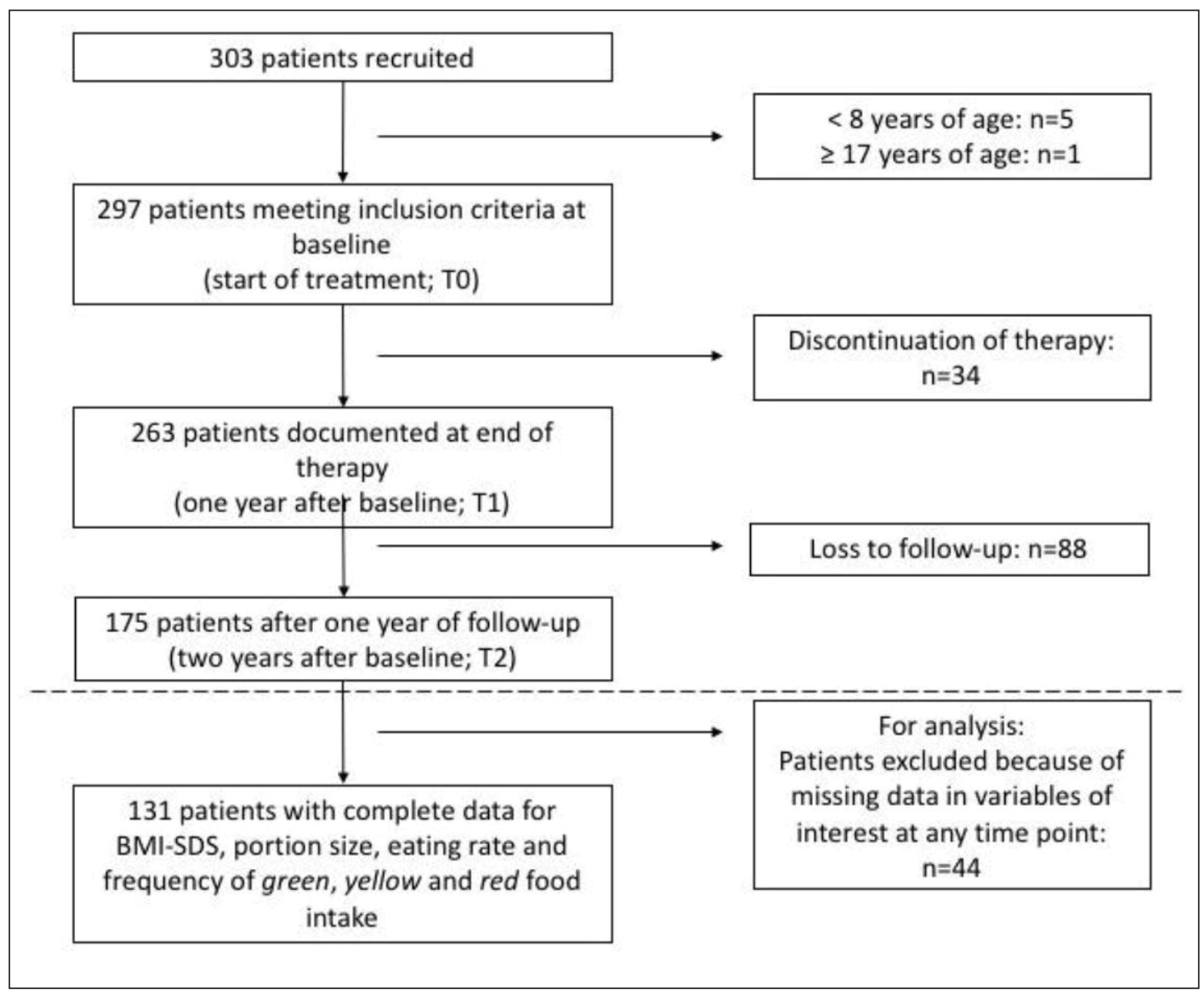

Fig. 1. Study flow-chart.

Baseline characteristics for all patients included in the study and patients with complete and incomplete data are presented in table 1 . At the start of the intervention, patients with complete data had a significantly lower BMI-SDS compared to those with incomplete data. The other characteristics did not differ significantly between both groups.

The change of BMI-SDS, portion size, eating rate as well as the food frequency items green, yellow and red intake are shown in table 2. BMI-SDS reduction was found to be significant for the 1- and 2-year period as well as for the reduction of portion size, eating rate and the intake of red food. The consumption of green food increased significantly only in the 1-year period while there was no significant 1- or 2-year change in the reported frequencies for yellow food items. The strongest 1- and 2-year effect size for changes in eating and nutritional behaviour was ascertained for the eating rate followed by portion size and frequencies for red food intake.

The 1-year reduction of BMI-SDS was positively associated with the reduction of portion size and eating rate and negatively with the food frequencies of green food items. The 2-year BMI-SDS change was associated significantly in favour of eating rate and portion size. Of the subscales assessing food quality only the change in frequency of green food item intake was negatively related to 1-year BMI-SDS reduction. It was also negatively associated with the one-year change in portion size (table 3). The regression model including the increase in consumption of green food items changed the estimate of the determinant of portion size for 1 -year BMI-SDS reduction by more than $10 \%$ and was regarded as the only potential confounder for the 1-year regressions. 
Torbahn et al.: Reduction of Portion Size and Eating Rate Is Associated with BMI-SDS Reduction in Overweight and Obese Children and Adolescents: Results on Eating and Nutrition Behaviour from the Observational KgAS Study

Table 1. Baseline characteristics of patients with complete and incomplete data

\begin{tabular}{|c|c|c|c|c|c|c|c|}
\hline \multirow[t]{2}{*}{$\begin{array}{l}\text { Name (unit or range) } \\
\text { of the respective scale }\end{array}$} & \multicolumn{2}{|c|}{$\begin{array}{l}\text { Total number of } \\
\text { patients at onset of } \\
\text { treatment }\end{array}$} & \multicolumn{2}{|c|}{$\begin{array}{l}\text { Follow-up with } \\
\text { complete data }\end{array}$} & \multicolumn{2}{|c|}{$\begin{array}{l}\text { Lost to follow-up } \\
\text { after } 1 \text { year, } \\
\text { incomplete data }\end{array}$} & \multirow[t]{2}{*}{$\mathrm{p}$ value } \\
\hline & $\mathrm{N}$ & mean $\pm \mathrm{SD}$ & $\mathrm{N}$ & mean \pm SD & $\mathrm{N}$ & mean \pm SD & \\
\hline BMI, $\mathrm{kg} / \mathrm{m}^{2}$ & 297 & $28.73 \pm 4.39$ & 131 & $28.15 \pm 4.01$ & 166 & $29.19 \pm 4.62$ & 0.08 \\
\hline BMI-SDS & 297 & $2.37 \pm 0.44$ & 131 & $2.30 \pm 0.39$ & 166 & $2.43 \pm 0.46$ & 0.01 \\
\hline Age, years & 297 & $11.74 \pm 1.86$ & 131 & $11.78 \pm 1.78$ & 166 & $11.71 \pm 1.93$ & 0.73 \\
\hline Female, \% & 154 & 51.85 & 68 & 51.91 & 86 & 51.81 & 0.99 \\
\hline SES, \% & 269 & & 122 & & 147 & & 0.39 \\
\hline Low & & 26.39 & & 30.33 & & 23.13 & \\
\hline Middle & & 52.05 & & 50.00 & & 53.74 & \\
\hline High & & 21.56 & & 19.67 & & 23.13 & \\
\hline Migration background, \% & 57 & 20.80 & 27 & 22.13 & 30 & 19.74 & 0.63 \\
\hline Portion size (1-7) & 282 & $4.18 \pm 1.16$ & 131 & $4.24 \pm 1.16$ & 151 & $4.12 \pm 1.16$ & 0.36 \\
\hline Eating rate $(0-100)$ & 283 & $65.43 \pm 17.22$ & 131 & $63.96 \pm 19.78$ & 152 & $62.83 \pm 18.36$ & 0.33 \\
\hline $\begin{array}{l}\text { Intake of sweets, snacks, fast food, } \\
\text { sausages, sweetened beverages, ice tea, } \\
\text { cocoa and fruit juices }(0-28)^{1}\end{array}$ & 284 & $19.81 \pm 13.37$ & 131 & $20.51 \pm 12.81$ & 153 & $19.21 \pm 13.84$ & 0.22 \\
\hline $\begin{array}{l}\text { Intake of cheese, milk, farmers cheese, } \\
\text { buttermilk, meat }(0-28)^{2}\end{array}$ & 284 & $11.80 \pm 7.68$ & 131 & $11.91 \pm 8.26$ & 153 & $11.71 \pm 7.18$ & 0.96 \\
\hline $\begin{array}{l}\text { Intake of fruits, vegetables, salads, } \\
\text { whole-grain bread }(0-28)^{3}\end{array}$ & 283 & $21.21 \pm 13.90$ & 131 & $21.98 \pm 14.15$ & 152 & $20.54 \pm 13.69$ & 0.30 \\
\hline
\end{tabular}

BMI-SDS = BMI standard deviation score; SES = socio-economic-status; SD = standard deviation.

$1,2,3$ Represents red, yellow and green food intake, respectively.

Table 2. Change in BMI-SDS and eating habits and food intake in patients with complete data $(\mathrm{n}=131)$

\begin{tabular}{|c|c|c|c|c|c|c|}
\hline \multirow[t]{2}{*}{$\begin{array}{l}\text { Name (range) of the } \\
\text { respective scale }\end{array}$} & \multicolumn{3}{|c|}{$\begin{array}{l}1 \text {-year change }(\mathrm{T} 1-\mathrm{T} 0) \text { in the } \\
\text { respective scale }\end{array}$} & \multicolumn{3}{|c|}{$\begin{array}{l}\text { 2-year change }(\mathrm{T} 2-\mathrm{T} 0) \text { in the } \\
\text { respective scale }\end{array}$} \\
\hline & $\Delta($ mean $\pm \mathrm{SD})$ & $\mathrm{p}$ value & Glass' $\Delta$ & $\Delta($ mean $\pm \mathrm{SD})$ & $\mathrm{p}$ value & Glass' $\Delta$ \\
\hline BMI-SDS & $-0.30 \pm 0.37$ & $<0.01$ & 0.77 & $-0.27 \pm 0.45$ & $<0.01$ & 0.69 \\
\hline Portion size (1-7) & $-0.67 \pm 1.11$ & $<0.01$ & 0.58 & $-0.54 \pm 1.16$ & $<0.01$ & 0.47 \\
\hline Eating rate $(0-100)$ & $-15.74 \pm 18.23$ & $<0.01$ & 0.80 & $-15.44 \pm 20.44$ & $<0.01$ & 0.78 \\
\hline $\begin{array}{l}\text { Intake of sweets, snacks, fast } \\
\text { food, sausages, sweetened } \\
\text { beverages, ice tea, cocoa } \\
\text { and fruit juices }(0-28)^{1}\end{array}$ & $-5.44 \pm 12.66$ & $<0.01$ & 0.42 & $-5.70 \pm 15.21$ & $<0.01$ & 0.44 \\
\hline $\begin{array}{l}\text { Intake of cheese, milk, } \\
\text { farmers cheese, } \\
\text { buttermilk and meat } \\
(0-28)^{2}\end{array}$ & $0.85 \pm 8.14$ & 0.1918 & 0.10 & $-0.8 \pm 8.43$ & 0.46 & 0.10 \\
\hline $\begin{array}{l}\text { Intake of fruits, vegetables, } \\
\text { salads, whole-grain bread } \\
(0-28)^{3}\end{array}$ & $6.51 \pm 15.92$ & $<0.01$ & 0.46 & $2.78 \pm 16.78$ & 0.10 & 0.20 \\
\hline
\end{tabular}


Torbahn et al.: Reduction of Portion Size and Eating Rate Is Associated with BMI-SDS Reduction in Overweight and Obese Children and Adolescents: Results on Eating and Nutrition Behaviour from the Observational KgAS Study

V 0

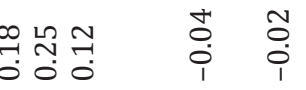

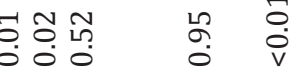

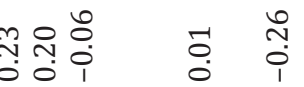

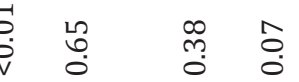

\begin{tabular}{llll}
$\infty$ & \multirow{0}{*}{} & $\infty$ & 0 \\
0 & 0 & 0 & 0 \\
0 & 1 & 0 & 1
\end{tabular}

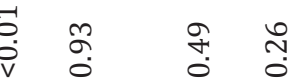

$\begin{array}{llll}\infty & \infty & 0 & 0 \\ & 0 & 0 & \stackrel{1}{0} \\ 0 & 1 & 0 & 1\end{array}$

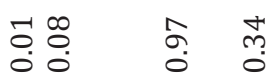

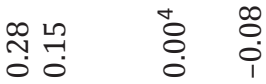

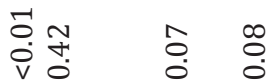

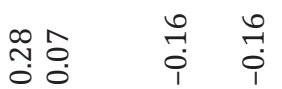
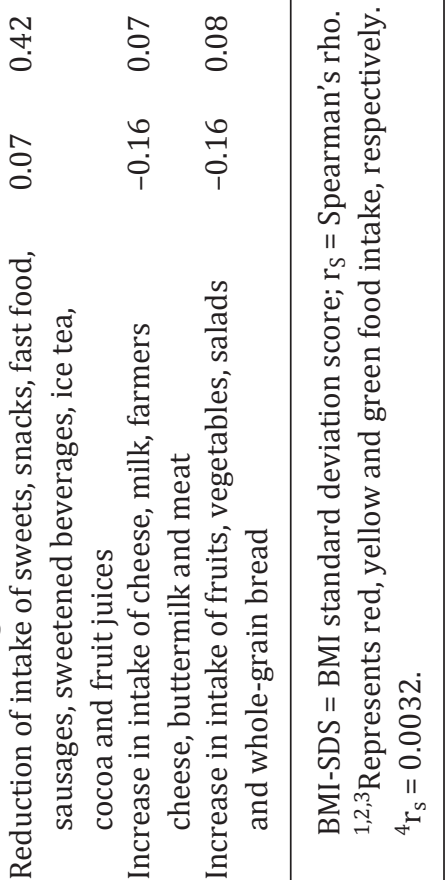
Torbahn et al.: Reduction of Portion Size and Eating Rate Is Associated with BMI-SDS Reduction in Overweight and Obese Children and Adolescents: Results on Eating and Nutrition Behaviour from the Observational KgAS Study

Table 4. Regression coefficients of reduction of portion size for 1- and 2-year BMI-SDS reduction using different models

\begin{tabular}{|c|c|c|c|c|c|c|}
\hline \multirow[t]{2}{*}{ Reduction of portion size } & \multicolumn{3}{|c|}{ 1-year BMI-SDS reduction } & \multicolumn{3}{|c|}{ 2-year BMI-SDS reduction } \\
\hline & $\mathrm{b} \pm \mathrm{SE}(95 \%-\mathrm{CI})$ & $\mathrm{p}$ value & Cohen's f ${ }^{2}$ & $\mathrm{~b} \pm \mathrm{SE}(95 \%-\mathrm{CI})$ & $\mathrm{p}$ value & Cohen's $\mathrm{f}^{2}$ \\
\hline Unadjusted & $\begin{array}{l}0.07 \pm 0.03 \\
(0.02 ; 0.13)\end{array}$ & 0.01 & & $\begin{array}{l}0.07 \pm 0.03 \\
(0.00 ; 0.14)^{4}\end{array}$ & 0.04 & \\
\hline $\begin{array}{l}\text { Adjusted for mandatory } \\
\text { confounders and } \\
\text { baseline values }^{1}\end{array}$ & $\begin{array}{l}0.16 \pm 0.04 \\
(0.09 ; 0.23)\end{array}$ & $<0.01$ & & $\begin{array}{l}0.14 \pm 0.04 \\
(0.06 ; 0.23)\end{array}$ & $<0.01$ & \\
\hline $\begin{array}{l}\text { Final model with further } \\
\text { adjustment of identified } \\
\text { confounders by changes } \\
\text { in dietary habits }{ }^{2,3}\end{array}$ & $\begin{array}{l}0.14 \pm 0.04 \\
(0.07 ; 0.22)\end{array}$ & $<0.01$ & 0.13 & $\begin{array}{l}0.14 \pm 0.04 \\
(0.06 ; 0.23)\end{array}$ & $<0.01$ & 0.09 \\
\hline
\end{tabular}

BMI-SDS = BMI standard deviation score; $b=$ regression coefficient; $\mathrm{SE}=$ standard error; $95 \% \mathrm{CI}=95 \%$ confidence interval; Cohen's $\mathrm{f}^{2}=$ effect sizes of 0.02 indicate small effects, 0.15 medium effects and 0.35 indicate large effects.

${ }^{1}$ BMI-SDS at baseline (T0), Portion size at baseline (T0), age, sex, SES, migration background.

${ }^{2}$ The only confounder for the 1-year reduction of portion size was increase of consumption of salad, fruits, vegetables, and whole-grain bread. No confounders were found for the 2-year period.

${ }^{3}$ For the final model regression coefficients and effect sizes remained significant are shown.

${ }^{4} 95 \%$ CI $(0.0045 ; 0.1365)$.

Association of the Reduction of Portion Size with 1- and 2-Year BMI-SDS Reduction

Compared to the unadjusted model, adjustment for age, sex, SES and migration background strengthened this association. After further adjustmentfor the increase in consumption of green food items, the association of change in portion size with 1-year BMI-SDS change was mitigated but remained significant (table 4).

\section{Association of the Reduction of Eating Rate with 1- and 2-Year BMI-SDS Reduction}

Calculated explanatory effects of the change of eating rate for both, the 1- and 2-year change were significant but small. Results of different regression models are shown in table 5. Comparing the regression coefficients of reduction of portion size (table 4) and reduction of eating rate (table 5), the reduction of portion size shows a higher association with 1- and 2 -year weight reduction. Effect sizes (Cohen's $\mathrm{f}^{2}$ ) were larger for eating rate. No interaction terms (for age, sex and SES) were significant.

\section{Discussion}

Both the reduction of portion size and eating rate were associated with 1- and 2-year BMI-SDS reduction. The results of this study also showed that a multimodal behavioural obesity treatment programme combining child and parental interventions can have a positive impact on eating and nutrition behaviours of children and adolescents. The participants of the study decreased their eating rates, portion sizes, and consumption of red foods in the 1and 2-year period. The effect sizes were moderate to high, which is reassuring given the fact that our intervention consists of different modules and the cognitive behavioural modification of portion size and eating rate are two important steps out of many, including physical activity. After conducting a comprehensive literature search we could not find any published 
Torbahn et al.: Reduction of Portion Size and Eating Rate Is Associated with BMI-SDS Reduction in Overweight and Obese Children and Adolescents: Results on Eating and Nutrition Behaviour from the Observational KgAS Study

Table 5. Regression coefficients of reduction of eating rate for 1- and 2-year BMI-SDS reduction using different models

\begin{tabular}{|c|c|c|c|c|c|c|}
\hline \multirow[t]{2}{*}{ Reduction of eating rate } & \multicolumn{3}{|c|}{ 1-year BMI-SDS reduction } & \multicolumn{3}{|c|}{ 2-year BMI-SDS reduction } \\
\hline & $\mathrm{b} \pm \operatorname{SE}(95 \%-\mathrm{CI})$ & p-value & Cohen's $\mathrm{f}^{2}$ & $\mathrm{~b} \pm \mathrm{SE}(95 \%-\mathrm{CI})$ & $\mathrm{p}$-value & Cohen's $\mathrm{f}^{2}$ \\
\hline Unadjusted & $\begin{array}{l}0.00 \pm 0.00 \\
(0.00 ; 0.01)^{4}\end{array}$ & 0.01 & & $\begin{array}{l}0.00 \pm 0.00 \\
(0.00 ; 0.01)^{6}\end{array}$ & $<0.05^{6}$ & \\
\hline $\begin{array}{l}\text { Adjusted for mandatory } \\
\text { confounders and } \\
\text { baseline values }^{1}\end{array}$ & $\begin{array}{l}0.01 \pm 0.00 \\
(0.01 ; 0.02)^{5}\end{array}$ & $<0.01$ & & $\begin{array}{l}0.01 \pm 0.00 \\
(0.00 ; 0.01)^{7}\end{array}$ & $<0.01$ & \\
\hline $\begin{array}{l}\text { Final model with } \\
\text { additional adjustment } \\
\text { by further changes in } \\
\text { eating and dietary } \\
\text { habits ascertained in } \\
\text { the study }{ }^{2,3}\end{array}$ & $\begin{array}{l}0.01 \pm 0.00 \\
(0.01 ; 0.02)^{5}\end{array}$ & $<0.01$ & 0.20 & $\begin{array}{l}0.01 \pm 0.00 \\
(0.00 ; 0.01)^{7}\end{array}$ & $<0.01$ & 0.10 \\
\hline
\end{tabular}

BMI-SDS = BMI standard deviation score; $b=$ regression coefficient; $\mathrm{SE}=$ standard error; $95 \%-\mathrm{CI}=95 \%$ confidence interval; Cohen's $\mathrm{f}^{2}=$ effect sizes of 0.02 indicate small effects, 0.15 medium effects and 0.35 indicate large effects.

${ }^{1}$ BMI-SDS at baseline (T0), eating rate at baseline (t0), age, sex, SES, migration background.

${ }^{2}$ The only further significantly change of nutritional behaviour that was associated with 1-year BMI-SDS reduction ascertained in the study was the higher frequency of consumption of salad, fruits, vegetables and whole-grain bread. There were no items found associated weight reduction for the 2-year period. No confounders were found for the 1- or 2-year period.

${ }^{3}$ For the final model regression coefficients and effect sizes remained significant are shown.

${ }^{4} 0.0046 \pm 0.0018(\mathrm{~b} \pm \mathrm{SE}), 95 \%$ CI $(0.0011 ; 0.0080)$.

${ }^{5} 0.0112 \pm 0.0021(\mathrm{~b} \pm \mathrm{SE}), 95 \%$ CI $(0.0070 ; 0.0154)$.

${ }^{6} 0.0038 \pm 0.0019(\mathrm{~b} \pm \mathrm{SE}), 95 \% \mathrm{CI}(0.0001 ; 0.0076), \mathrm{p}$ value 0.0454 .

${ }^{7} 0.0082 \pm 0.0024(\mathrm{~b} \pm \mathrm{SE}), 95 \%$ CI $(0.0035 ; 0.0129)$.

data for associations between changes of portion size and eating rate from interventional or longitudinal studies for children and adolescents.

The results for BMI-SDS reduction in this analysis (complete data for BMI-SDS and nutritional variables) are comparable to the results that have been published before (complete data only for BMI-SDS) [32].

We show a significant positive association between the reduction of portion size and 1and 2-year weight reduction. Results of mediator analyses of evaluations of treatment programmes for adults showed also a significant association of the reduction of portion size with weight loss [33,34]. In our study, the effect sizes of this association were between small and medium (Cohen's $\mathrm{f}^{2}=0.13$ and Cohen's $\mathrm{f}^{2}=0.09$ ). This is in line with results from a recently published Cochrane review, which revealed that for adults the effect achieved by interventions reducing portion size alone would be too small for achieving the targets of weight loss recommended by the UK National Institute for Health and Care and should be supported by further intervention modules (such as movement behaviour) [12].

Furthermore, the questionnaire for measuring portion size was newly developed. We used elements (pictures) of the validated instrument YANA-C that was computer based, extended the measurement of portion sizes to meals other than pasta (e.g. French fries, breakfast cereals) and designed for self-report of food intake in adolescents [24, 25]. Not only the methods but also the purpose of our assessment differed as our instrument sought to estimate unobserved portion size without parental supervision or the presence of peers. 
Torbahn et al.: Reduction of Portion Size and Eating Rate Is Associated with BMI-SDS Reduction in Overweight and Obese Children and Adolescents: Results on Eating and Nutrition Behaviour from the Observational KgAS Study

Additionally, it could be influenced by a combination of the distensibility / physical size of the stomach (which in turn is influenced by habitual portion size), attention to signals of fullness and satiety, eating rate (as it affects the feedback signal) and self-control of portion size.

We could identify small or medium effects for the reduction of eating rate on 1- and 2 -year weight reduction (Cohen's $\mathrm{f}^{2}=0.20$ and Cohen's $\mathrm{f}^{2}=0.10$ ). The effect sizes of 1 - and 2-year changes of eating rate on BMI-SDS reduction were higher compared to the changes of portion size. A better validity of the eating rate instrument (FKE-KJ) could be one explanation. Provided that validities are comparable, patients would profit more from strategies to reduce eating rate than portion size. We did not find any previously published longitudinal correlational studies of eating rates and obesity. Only one (pilot) study was found that reported data on the change of eating rates (by the use of device that monitors patient's eating rate), but its targets have not been reached [35]. For the association of eating rate and obesity, a meta-analysis of 23 studies revealed a mean difference in BMI of $1.78 \mathrm{~kg} / \mathrm{m}^{2}$ (range 1.53-2.04 $\mathrm{kg} / \mathrm{m}^{2}$ ) between those with a high and low eating rate [36].

Amongst the variables of nutrition behaviour, only the change in consumption of food in the green items was associated with 1-year BMI-SDS change.

We showed significant changes in the intake of red food items for the 1- and 2-year period, while the intake of green food items was significant only for the 1-year period. Two studies were identified that described changes of food intake after a multidisciplinary behaviour-based patient education programme. In line with our results, Epstein et al. [37] showed significant 1- and 2-year changes for red and green food. Adam et al. [38] reported similar results. As differences between self-reported and parent-reported estimations are to be expected, it is important to mention that Epstein et al. [37] assessed food intake by a combination of parent- and patient-report and Adam et al. [38] used self-reported FFQs, while we used a parent-reported FFQ [39]. We found that a higher intake of green food was associated with 1-year weight reduction in children and adolescents. A more frequent intake of vegetables and fruits was found to be associated with higher weight reduction for a longterm period in children and adolescents, while there was no effect on weight loss in adults $[37,40]$. Nevertheless, a more frequent intake of vegetables, fruits and whole-grain products is important for a healthy level of vitamins and minerals and should also be targeted in interventions. We could not show any change of the frequency of yellow food intake what is in line with the results of Epstein et al [37]. A possible explanation could be that this group consists of a higher diversity of food (milk, dairy products, eggs and meat). Therefore, changes could be possible in both directions (e.g. while patients reduced the intake of meat they, increased the consumption of milk). While such interventions impart patients to reduce intake of red food and increase intake of green food, for the yellow group it is more important to reduce fat rate (e.g. change from full-cream milk to semi-skimmed milk). This change was not assessed in our study. We also did not find any effect of an intake reduction in red food items on 1- or 2-year BMI-SDS change. This could be due to the parents-reported red food items, since children and adolescents might consume favoured high-energy-dense foods without the knowledge of their parents. Moreover, parents may feel themselves urged to report an optimised intake of their children as they feel responsible for their children's nutrition. Due to cost restraints and because of a lack of valid and feasible alternatives, FFQs are one of the most used instruments to assess food intake in observational studies despite its shortcomings $[41,42]$. Nutrition behaviour by self-report is not only fraught with errors of recall but also influenced by false reporting in a socially acceptable direction (usually underreporting of energy-dense foods) or wishful thinking and denial $[43,44]$. We therefore chose to use the reporting of nutrition by the parents.

For the interpretation of our findings, some strengths and limitations have to be considered. 
Torbahn et al.: Reduction of Portion Size and Eating Rate Is Associated with BMI-SDS Reduction in Overweight and Obese Children and Adolescents: Results on Eating and Nutrition Behaviour from the Observational KgAS Study

To our knowledge, this is the first work that reports results on the change of portion size and eating rate and their effect on BMI-SDS reduction after a guideline-orientated multidisciplinary conservative patient education programme for overweight and obese children and adolescents [17]. Furthermore, food intake and eating rate were assessed by validated instruments. A further strength is the sample size of 297 patients that could be included in the study.

Our restriction to complete follow-up data (ignoring participants lost to follow-up) and the significant difference of BMI-SDS between patients with complete and incomplete data at baseline could result in bias and therefore in overestimated effect estimates for behavioural changes. The rate of loss to follow up was high (around 30\%) but is comparable to other trials [5]. Patients lost to follow-up 1 year after the end of treatment showed a smaller 1-year BMI-SDS reduction and behaviour change [32]. The associations between behaviour change and BMI-SDS change which is the focus of our study should, however, be unaffected by selection bias due to loss to follow-up. A controlled comparative study would likely have increased the validity of the results. Due to ethical, organisational and financial limitations, we were unable to perform a study with an untreated control arm [32]. Due to the fact that inclusion and exclusion criteria of our intervention are based and comparable to the recommendations in the German guideline, this sample of patients is representative for children and adolescents treated in multimodal programmes [17]. Patient characteristics such as age, sex, migration background, BMI-SDS at baseline could differ at time of inclusion in treatment programmes. Therefore, results of reported effects could differ when comparing studies reporting results from such (comparable) treatments.

While data on BMI-SDS is based on measurements, all other outcomes have been assessed via questionnaires. The true influence of behaviour change, and especially nutrition behaviour, may be underestimated. This may be especially true for the measurement of nutrition behaviour as the assessment of food intake is fraught with limitations in validity. Furthermore, as mentioned above, the instrument for measuring portion size was newly developed, and therefore results should be interpreted with caution. Further studies should use validated instruments for the assessment of portion size. We did not collect data on parent's diet or body weight. How these interventions impact the parents' health status and diet should be assessed and addressed in further research.

\section{Conclusion}

This present study shows that a reduction of portion size and eating rate is associated with 1- and 2-year BMI-SDS reduction after an out-patient, multidisciplinary, conservative patient education programme for overweight and obese children and adolescents. The findings support to focus on appropriate portion sizes and reduced eating rate as part of patient and parent training for overweight and obese children and adolescents. For further research, the assessment, evaluation and reporting of change of portion size and eating rate assessed by standardised instruments is needed.

\section{Acknowledgements}

We like to thank all professionals from the study centres and all families and patients who were involved in this study. 


\section{Financial Support}

This study received financial support from the BZgA and the AID Infodienst. Furthermore, the Barmer GEK bore the costs for the treatment programme for all their patients by a binding information $(10 \%$ of patients participated in the study).

\section{Authors' Contributions}

Conceived and designed the study: AvE, RWH. Analysed the data: GT, AvE, KF. Wrote the first draft of the manuscript: GT. Contributed to the writing of the manuscript: GT, AvE, IG, BK, RvK, VO, KF and RWH. All authors (GT, IG, BK, RvK, VO, RWH, AvE) reviewed the manuscript critically for important intellectual content. All authors read and approved the final manuscript.

\section{Ethical and Legal Prerequisites}

The trial protocol has been approved by the ethical committee of the University of Ulm.

\section{Disclosure Statement}

GT, AvE, IG, BK work unsalaried for the KgAS society. IG, AvE, BK, KF, and RWH received fees and/or travel costs from AID Infodienst. GT and BK are team members in out-patient centres that are certified by KgAS society.

\section{References}

1 Ezzati M, Riboli E: Behavioral and dietary risk factors for noncommunicable diseases. N Engl J Med 2013;369: 954-964.

2 Simmonds M, Llewellyn A, Owen CG, Woolacott N: Predicting adult obesity from childhood obesity: a systematic review and meta-analysis. Obes Rev 2016;17:95-107.

3 Kurth BM, Schaffrath Rosario A: Die Verbreitung von Übergewicht und Adipositas bei Kindern und Jugendlichen in Deutschland. Bundesgesundheitsbl Gesundheitsforsch Gesundheitsschutz 2007;50:736-743.

4 Brettschneider AK, Schaffrath Rosario A, Kuhnert R, Schmidt S, Wiegand S, Ellert U, Kurth BM: Updated prevalence rates of overweight and obesity in 11- to 17-year-old adolescents in Germany. Results from the telephone-based KiGGS Wave 1 after correction for bias in self-reports. BMC Public Health 2015;15:1101.

5 Mühlig Y, Wabitsch M, Moss A, Hebebrand J: Weight loss in children and adolescents. Dtsch Arztebl Int 2014; 111:818-824.

6 Mead E, Brown T, Rees K, Azevedo LB, Whittaker V, Jones D, Olajide J, Mainardi GM, Corpeleijn E, O'Malley C, Beardsmore E, Al-Khudairy L, Baur L, Metzendorf M-I, Demaio A, Ells LJ. Diet, physical activity and behavioural interventions for the treatment of overweight or obese children from the age of 6 to 11 years. Cochrane Database of Syst Rev 2017;6:CD012651.

7 Al-Khudairy L, Loveman E, Colquitt JL, Mead E, Johnson RE, Fraser H, Olajide J, Murphy M, Velho RM, O'Malley C, Azevedo LB, Ells LJ, Metzendorf M-I, Rees K. Diet, physical activity and behavioural interventions for the treatment of overweight or obese adolescents aged 12 to 17 years. Cochrane Database of Syst Rev 2017; 6:CD012691.

8 Kelley GA, Kelley KS, Pate RR: Exercise and BMI in overweight and obese children and adolescents: a systematic review and trial sequential meta-analysis. Biomed Res Int 2015;2015:704539.

9 Hummel E, Wittig F, Schneider K, Gebhardt N, Hoffmann I: The complex interaction of causing and resulting factors of overweight/obesity. Increasing the under-standing of the problem and deducing requirements for prevention strategies. Ernaehrungs Umschau Int 2013;60:2-7.

10 Spiegelman BM, Flier JS: Obesity and the regulation of energy balance. Cell;104:531-543.

11 Rouhani MH, Haghighatdoost F, Surkan PJ, Azadbakht L: Associations between dietary energy density and obesity: a systematic review and meta-analysis of observational studies. Nutrition;32:1037-1047.

12 Hollands GJ, Shemilt I, Marteau TM, Jebb SA, Lewis HB, Wei Y, Higgins JP, Ogilvie D: Portion, package or tableware size for changing selection and consumption of food, alcohol and tobacco. Cochrane Database Syst Rev 2015;1:CD011045. 
Torbahn et al.: Reduction of Portion Size and Eating Rate Is Associated with BMI-SDS Reduction in Overweight and Obese Children and Adolescents: Results on Eating and Nutrition Behaviour from the Observational KgAS Study

13 Young LR, Nestle M: Reducing portion sizes to prevent obesity: a call to action. Am J Prev Med 2012;43:565568.

14 Kling SM, Roe LS, Keller KL, Rolls BJ: Double trouble: Portion size and energy density combine to increase preschool children's lunch intake. Physiol Behav 2016;162:18-26.

15 Young LR, Nestle M: Portion sizes and obesity: responses of fast-food companies. J Public Health Policy 2007; 28:238-248.

16 Almiron-Roig E, Tsiountsioura M, Lewis HB, Wu J, Solis-Trapala I, Jebb SA: Large portion sizes increase bite size and eating rate in overweight women. Physiol Behav 2015;139:297-302.

17 Wabitsch M, Kunze D: Konsensbasierte (S2) Leitlinie zur Diagnostik, Therapie und Prävention von Übergewicht und Adipositas im Kindes- und Jugendalter. Version 15.10.2015. www.aga.adipositas-gesellschaft.de/ fileadmin/PDF/Leitlinien/AGA_S2_Leitlinie.pdf (last accessed October 6, 2017).

18 Gellhaus I, Eberding A, Gruber W, Jaeschke R, Kühn-Dost A, Stübing K, Tiedjen U, Weidanz I, Werning A: Adipositasschulungsprogramm «Leichter, aktiver, gesünder» - Programm-Handbuch. Konsensusgruppe Adipositasschulung im Kindes- und Jugendalter e. V. (KgAS), 2009.

19 Stachow R, Stübing K, van Egmond-Fröhlich A, Vahabzadeh Z, Jaeschke R, Kühn-Dost A, Eberding A, Westenhöfer J, Mannhardt S, Ernst M, Claußnitzer G, Lawrenz A, Lob-Corzilius T, Mast M, Tiedjen U, Wabitsch M: Trainermanual: «Leichter, aktiver, gesünder». Interdisziplinäres Konzept für die Schulung übergewichtiger oder adipöser Kinder und Jugendlicher. aid Infodienst, Deutsche Gesellschaft für Ernährung (DGE), Konsensusgruppe Adipositasschulung im Kindes- und Jugendalter (KgAS), 2004.

20 Cole TJ, Green PJ: Smoothing reference centile curves: the LMS method and penalized likelihood. Stat Med 1992;11:1305-1319.

21 Kromeyer-Hauschild K, Moss A, Wabitsch M: Referenzwerte für den Body-Mass-Index für Kinder, Jugendliche und Erwachsene in Deutschland. Anpassung der AGA-BMI-Referenz im Altersbereich von 15 bis 18 Jahren. Adipositas 2015;9:123-127.

22 Warschburger P, Petermann F: Fragebogen zum konkreten Essverhalten (FKE-KJ); Adipositas. Göttingen, Hogrefe, 2007.

23 Warschburger P, van Egmond-Fröhlich A, Hudjetz A, Döring I, Kröller K, Kühne D, Baudach A, Claußnitzer G, Fiedler S, Oepen J, Waldeck E, Stübing K, Haerting J: Einfluss eines Elterntrainings auf die Gewichtsentwicklung adipöser Grundschulkinder: Die EPOC-Studie: Jahrestagung der Deutschen Gesellschaft für Kinder- und Jugendmedizin. Bielefeld, 2011.

24 Vereecken CA, Covents M, Matthys C, Maes L: Young adolescents' nutrition assessment on computer (YANA-C). Eur J Clin Nutr 2005;59:658-667.

25 Vereecken C, Dohogne S, Covents M, Maes L: How accurate are adolescents in portion-size estimation using the computer tool Young Adolescents' Nutrition Assessment on Computer (YANA-C)? Br J Nutr 2010;103: 1844-1850.

26 Winkler J, Stolzenberg H: Der Sozialschichtindex im Bundes-Gesundheitssurvey. Gesundheitswesen 1999; 61(suppl 2):S178-S183.

27 Lampert T, Kurth B-M: Sozialer Status und Gesundheit von Kindern und Jugendlichen: Ergebnisse des Kinderund Jugendgesundheitssurveys (KiGGS). Dtsch Arztebl Int 2007;6:521-526.

28 Hoffmeister U, Bullinger M, van Egmond-Fröhlich A, Goldapp C, Mann R, Ravens-Sieberer U, Reinehr T, Westenhöfer J, Holl R: Beobachtungsstudie der BZgA zur Adipositastherapie bei Kindern und Jugendlichen in Deutschland: Anthropometrie, Komorbidität und Sozialstatus. Klin Padiatr 2010;222:274-278.

29 Hedges LV, Olkin I: Statistical Methods for Meta-Analysis. New York, Academic Press, 1982.

30 Cohen J: Statistical Power Analysis for the Behavior Science, 2nd ed. New York, Academic Press, 1988.

31 R Core Team. R: A language and environment for statistical computing, R Foundation for Statistical Computing, Vienna, 2015,

32 Gellhaus I, van Egmond-Fröhlich A, Tiedjen U, Koch B, Torbahn G, Flothkötter M, Fink K, Holl R: Gewicht, Gesundheit, Lebensqualität. Ergebnisse der KgAS-Adipositasschulung bei Kindern und Jugendlichen in Deutschland. Adipositas 2016;10:4-10.

33 Coughlin JW, Gullion CM, Brantley PJ, Stevens VJ, Bauck A, Champagne CM, Dalcin AT, Funk KL, Hollis JF, Jerome GJ, Lien LF, Loria CM, Myers VH, Appel LJ: Behavioral mediators of treatment effects in the weight loss maintenance trial. Ann Behav Med 2013;46:369-381.

34 Young MD, Lubans DR, Collins CE, Callister R, Plotnikoff RC, Morgan PJ: Behavioral mediators of weight loss in the SHED-IT community randomized controlled trial for overweight and obese men. Ann Behav Med 2015;49: 286-292.

35 Hamilton-Shield J, Goodred J, Powell L, Thorn J, Banks J, Hollinghurst S, Montgomery A, Turner K, Sharp D: Changing eating behaviours to treat childhood obesity in the community using Mandolean: the Community Mandolean randomised controlled trial (ComMando) - a pilot study. Health Technol Assess 2014;18:1-75.

36 Ohkuma T, Hirakawa Y, Nakamura U, Kiyohara Y, Kitazono T, Ninomiya T: Association between eating rate and obesity: a systematic review and meta-analysis. Int J Obes (Lond) 2015;39:1589-1596.

37 Epstein LH, Paluch RA, Beecher MD, Roemmich JN: Increasing healthy eating vs. reducing high energy-dense foods to treat pediatric obesity. Obesity (Silver Spring) 2008;16:318-326.

38 Adam S, Westenhofer J, Rudolphi B, Kraaibeek HK: Effects of a combined inpatient-outpatient treatment of obese children and adolescents. Obes Facts 2009;2:286-293. 
Torbahn et al:: Reduction of Portion Size and Eating Rate Is Associated with BMI-SDS Reduction in Overweight and Obese Children and Adolescents: Results on Eating and Nutrition Behaviour from the Observational KgAS Study

39 Burrows TL, Truby H, Morgan PJ, Callister R, Davies PS, Collins CE: A comparison and validation of child versus parent reporting of children's energy intake using food frequency questionnaires versus food records: who's an accurate reporter? Clin Nutr 2013;32:613-618.

40 Kaiser KA, Brown AW, Bohan Brown MM, Shikany JM, Mattes RD, Allison DB: Increased fruit and vegetable intake has no discernible effect on weight loss: a systematic review and meta-analysis. Am J Clin Nutr 2014; 100:567-576.

41 Shim JS, Oh K, Kim HC: Dietary assessment methods in epidemiologic studies. Epidemiol Health 2014; 36:e2014009.

42 Willett W: Foreword. The validity of dietary assessment methods for use in epidemiologic studies. Br J Nutr 2009;102(suppl 1):S1-2.

43 Bandini LG, Schoeller DA, Cyr HN, Dietz WH: Validity of reported energy intake in obese and nonobese adolescents. Am J Clin Nutr 1990;52:421-425.

44 Lanfer A, Hebestreit A, Ahrens W: Diet and eating habits in relation to the development of obesity in children and adolescents. Bundesgesundheitsbl Gesundheitsforsch Gesundheitsschutz 2010;53:690-698. 